\title{
SPECTRAL SENSITIVITY AND CONVERGENCE RATE IN ADAPTIVE IIR FILTERING
}

\author{
José A. RODRIGUEZ-FONOLLOSA and Enrique MASGRAU \\ Departament de Teoria del Senyal i Comunicacions \\ Universitat Politècnica de Catalunya \\ Apdo. 30.002, 08080 Barcelona, Spain.
}

It is known that, in the autoregressive model the spectral sensitivity curves are almost flat for the log-area ratios and in this paper it is shown that this property can be useful in adaptive IIR filtering. A new IIR lattice-form algorithm for adaptive filtering is proposed in which the update equation is applied to these parameters instead of the reflection coefficients. This transformation gives a more uniform performance surface with no unstability region and, as a result, the behavior of the Gauss Newton search method is clearly improved.

\section{Introduction}

To date, the majority of adaptive filtering problems have been solved with finite-impulseresponse (FIR) filters because they are well behaved, i.e., they are unconditionally stable and their error surface is unimodal. However, infiniteimpulse-response (IIR) filters present some advantages over FIR filters and it is anticipated that the adaptive IIR filter will replace the widely-used adaptive FIR filter in many applications.

Adaptive IIR filtering has been an active area of research over the last several years and it is also closely related to the problem of recursive parameter estimation. Nevertheless several problems still remain unsolved because the analysis involves highly nonlinear systems. Unlike adaptive FIR filtering, the error surfaces for adaptive IIR filters based on the output error formulation [1] may have some local minima. Their convergence properties are also difficult to study and computer simulations are usually necessary to compare the performance of different algorithms.

This work was supported by PRONTIC grant $N^{2} 105 / 88$
In adaptive FIR filtering the performance or error surface, (excess squared error), is a quadratic function with respect to the coefficients and gradient techniques perform well because there is a linear relation between the coefficients vector error and the gradient. However, in adaptive IIR filtering the error surfaces are very different and the slow rate of convergence which is characteristic of this class of adaptive algorithms is in part due to this nonuniform performance surface. Another drawback associated with the IIR filters is that they may become unstable during adaptation and it is usually necessary to include a stability test. The proposed algorithm has been derived with the aim of solving or reducing these two problems.

\section{IJR Lattice form}

The adaptive IIR lattice filter (Fig. 1) consists of a feedback lattice structure characterized by the reflection coefficients $\left\{k_{i}(n)\right\}$ and a feedforward structure characterized by the coefficients $\left\{v_{i}(n)\right\}$ [1]. Its primary advantage is that stability monitoring is easy to perform, requiring only that each reflection coefficient satisfy $\left|k_{i}(n)\right|<1$.

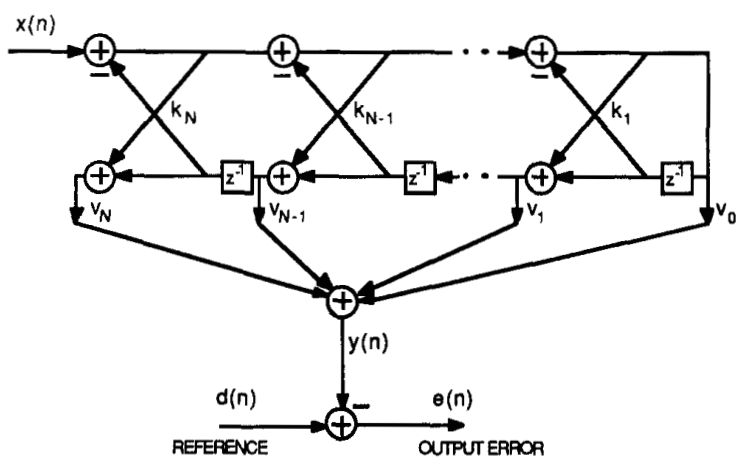

Figure 1. IIR lattice filter 
The forward and backward residuals of each stage are calculated as

$$
\begin{aligned}
& f_{N}(n)=x(n) \\
& f_{i-1}(n)=f_{i}(n)-k_{i}(n) b_{i-1}(n-1) \\
& b_{i}(n)=b_{i-1}(n-1)+k_{i}(n) f_{i-1}(n) \\
& b_{0}(n)=f_{0}(n)
\end{aligned}
$$

where $x(n)$ is the filter input.

Then the output $y(n)$ is obtained as the sum of the backward residuals weighted by the feedforward coefficients

$$
y(n)=\sum_{i=0}^{N} b_{i}(n) v_{i}(n)
$$

The lattice implementation need more computations than the direct form but it has better limited precision properties. The direct form is very sensitive to finite-precision effects so other realizations are usually preferred in fixed filter implementations. In adaptive applications these alternative realizations has been somewhat overlooked because of the simplicity of the direct form but it has been shown recently that structures as the parallel, cascade, and lattice can be useful to overcome the limited precision sensitivity and they offer more simple stability monitoring.

\section{Adaptive IIR algorithms}

Most of the adaptive algorithms are gradientbased methods, i.e., algorithms designed to minimize at each instant of time a cost function. This methods perform well when there is a linear relation between the coefficients vector error and the gradient as in FIR filtering.

Nevertheless, in adaptive IIR filtering, this kind of algorithms usually have convergence and stability problems. One of the reasons of these problems is that the gradient tends to infinite when the coefficients are close to the unstability region [2]. In the IIR lattice filter is easy to know how far we are from this unstability region and in [2] a correction term was derived to reduce the absolute value of the gradient used by the adaptive algorithm as the coefficients get close to the limits of the stability region. The result was a significant improvement in both convergence and robustness of the IIR steepest descent algorithms.
In the work that we present here we have followed another approach in which the performance surface of the adaptive algorithm is modified by a coefficient transformation based on spectral sensitivity. In [3] it is shown that in the all-pole model the sensitivity curves are almost flat for the logarithms of the ratios of area (log-area-ratios). Therefore, it is expected that if we express the mean squared output error as a function of the logarea ratios instead of the reflection coefficients, the error surface will be closer to the desired quadratic shape. Another advantage of this transformation is that now there is no unstability region.

The algorithms considered in this study are based on the Gauss-Newton (GN) search method described in [4]. In this kind of algorithms the filter parameters are updated by the following equation

$$
\theta(n+1)=\theta(n)-\mu H^{-1}(n) \psi(n) e(n)
$$

where $\theta(n)$ is the coefficient vector

$$
\theta(n)=\left[l_{1}(n), \ldots, I_{N}(n), v_{0}(n), \ldots, v_{N}(n)\right]
$$

and $e(n)$ the output error. $\psi(n)$ is the gradient vector formed with the the derivative of the output of the adaptive filter $y(n)$ with respect to the coefficients

$$
\psi(n)=\left[\frac{\partial y(n)}{\partial I_{1}(n)}, \ldots, \frac{\partial y(n)}{\partial I_{N}(n)}, \frac{\partial y(n)}{\partial v_{1}(n)}, \ldots, \frac{\partial y(n)}{\partial v_{N}(n)}\right]
$$

The scalar $\mu$ is the step size and $\mathrm{H}^{-1}$ is an estimate of the inverse Hessian matrix. To avoid the inversion of $H$, its inverse is updated directly using the matrix inversion lemma

$$
\begin{aligned}
& H^{-1}(n+1)= \\
& \frac{1}{\lambda}\left(H^{-1}(n)-\frac{H^{-1}(n) \psi(n) \psi^{T}(n) H^{-1}(n)}{\lambda / \mu+H^{-1}(n) \psi(n) \psi^{T}(n) H^{-1}(n)}\right)
\end{aligned}
$$

where $\lambda=1-\mu$. The Hessian matrix modifies the search direction and gives algorithms with improved rate convergence at the expense of increased computational complexity. In this paper we have considered algorithms using the full Hessian matrix but it is expected that the results can be also extended to other algorithms with simplified Hessians. 
In adaptive IIR filtering the Hessian matrix depends only on the data but in adaptive IIR filtering it is time-varying even when the data is stationary because the error surface is not quadratic. Again, a error surface close to the quadratic shape can be useful to obtain a less time-varying and better estimated Hessian matrix.

A major drawback of the lattice realization is that the computation of the gradient is quite more complicated than that of the direct form requiring order $\mathrm{N}^{2}$ computations compared to order $\mathrm{N}$ [5], and no satisfactory simplified form has been derived. Nevertheless our current work indicates that it is possible to reduce considerably the computation cost and we have also derived new simplified gradient expressions [6].

\section{LAR-GN Algorithm}

In the algorithms studied in [4] the parameters $l_{i}(n)$ were the reflection coefficients $k_{i}(n)$ while in the proposed algorithm these coefficients are replaced by the log-area-ratio parameters (LAR).

The log-area-ratios $l_{i}$ are calculated from the reflection coefficients $k_{i}$ as

$$
l_{i}=\ln \frac{1-k_{i}}{1+k_{i}}
$$

In the gradient vector the derivative of $y(n)$ with respect to the log-area-ratio is calculated now as

$$
\begin{aligned}
\frac{\partial y(n)}{\partial l_{i}(n)} & =\frac{\partial y(n)}{\partial k_{i}(n)} \frac{\partial k_{i}(n)}{\partial l_{i}(n)} \\
& =\frac{\partial y(n)}{\partial k_{i}(n)}\left\{-\frac{1}{2}\left(1-k_{i}(n)^{2}\right\}, \quad i=1 \cdot \cdot N\right.
\end{aligned}
$$

Then, after updating the log-area-ratio parameters with equation (3), the reflection coefficients must be computed to be used by the adaptive IIR lattice filter.

$$
k_{i}=\frac{1-e^{l_{i}}}{1+e^{l_{i}}}, \quad i=1 \cdot \cdot N
$$

In a real-time implementation the above equation can be simplified and stored in a table to reduce the computation cost.

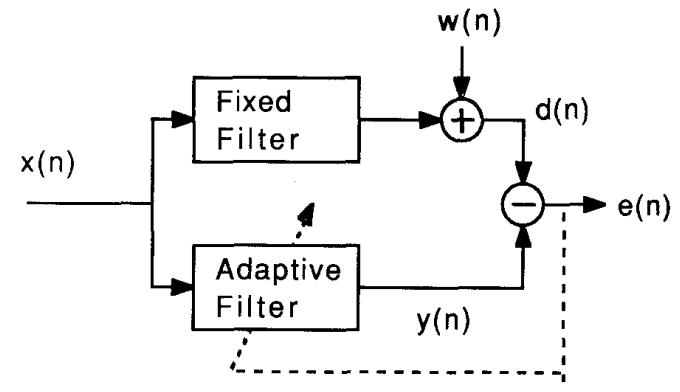

Figure 2. System Identification Configuration

\section{Computer Simulations}

We have performed several computer simulations in a system identification configuration (Fig. 2) to compare the convergence properties of the proposed algorithm (LAR-GN) with the previous full Hessian algorithm (K-GN) described in [4]. Figure 3 shows the mean-square-error learning curves that were obtained by averaging $e^{2}(n)$ over 1000 independent computer runs. For both algorithms $\mu=0.015$ gives the best results. The system to be identified has the following lattice coefficients

$$
\begin{array}{lllll}
v_{0}=1 & v_{1}=0 & v_{2}=0 & v_{3}=0 & v_{4}=0 \\
k_{1}=-0.91 & k_{2}=0.60 & k_{3}=-0.20 & k_{4}=0.1 &
\end{array}
$$

The convergence rate of the proposed algorithm (LAR-GN) is clearly faster than that of the (K-GN). This results was also obtained when the algorithm was tested with other systems and the difference between both algorithms was more clear when the unknown system had the poles near of unity circle.

Figure 4 shows the $k_{1}$ coefficient track for both algorithms in a system identification case of second order. The parameters of the unknown system are now

$$
\begin{array}{lll}
v_{0}=1 & v_{1}=0 & v_{2}=0 \\
k_{1}=0.94 & k_{2}=0.6 &
\end{array}
$$

Observe that the proposed algorithm converges more rapidiy and with less noisy track. In this case $\mu=0.015$ is still a good value for the LAR-GN algorithm but it must reduced to $\mu=0.004$ in the $K$ $\mathrm{GN}$ to avoid convergence problems. 


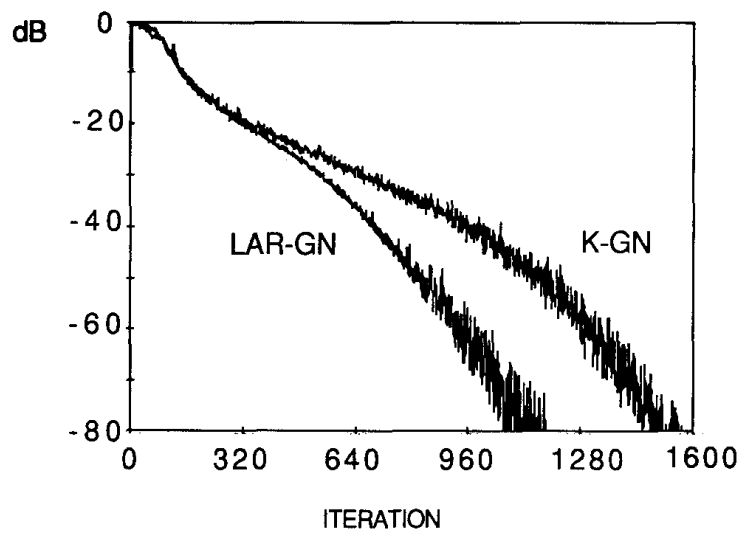

Figure 3. MSE learning curves

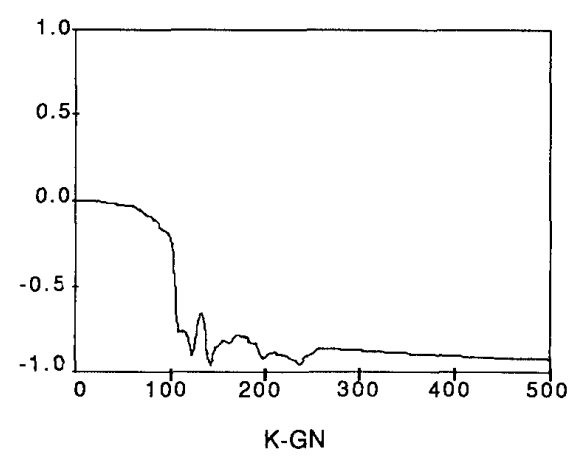

Figure 4a. K-GN coefficient track

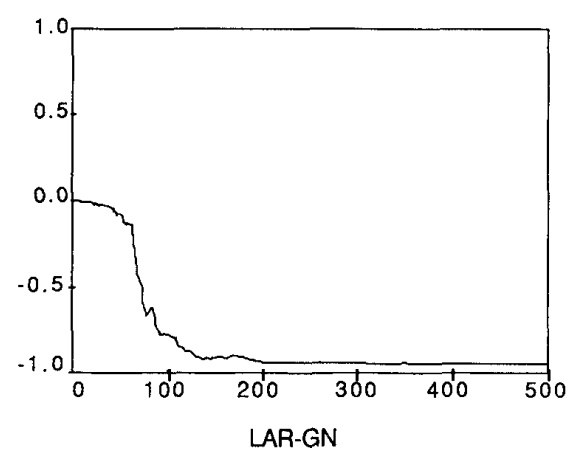

Figure 4b. LAR-GN coefficient track

\section{Conclusions}

A new algorithm for Adaptive IIR lattice filtering has been derived in which the log-area ratio parameters are updated instead of the reflection coefficients. This modification gives an error surface with no unstability region and with a more uniform behavior. Effectively, the results show that the application of the Gauss-Newton method to the log-area-ratio parameters gives an algorithm with a convergence rate faster than any other IIR adaptive algorithm.

We are currently working in a simplified IIR lattice gradient that will make this structure more competitive in computational cost.

\section{References}

[1] John J. Shynk. "Adaptive IIR Filtering". IEEE ASSP mag., Vol. 6, No. 2 April 1989

[2] José A. Rodríguez - Fonollosa. "Improved Convergence of Gradient algorithms for Adaptive IIR Filters". IEEE Proc. ISCAS'89, pp 1732-1735, May 1989.

[3] R. Viswanathan, J. Makhoul. "Quantization Properties of Transmission Parameters in Linear Predictive Systems". IEEE Trans. on ASSP, Vol 23, pp 309-321. June 1975.

[4] John J. Shynk. "On Lattice-form Algorithms for Adaptive IIR filtering". IEEE Proc. ICASSP, pp 1554-1557. 1988 .

[5] John J. Shynk. "Performance of alternative adaptive IIR realization". Proc. 21st Asilomar Conf. Signals, Systems and Computers, Pacific Gorve, CA, Nov. 1976.

[6] José A. Rodríguez-Fonollosa. "An Adaptive IIR Lattice Algorithm with simplified gradient calculation". (In preparation). 